\title{
Gradient flow and scale setting for twisted mass fermions
}

\author{
Albert Deuzeman* and Urs Wenger \\ Institute for Theoretical Physics \\ Albert Einstein Center for Fundamental Physics \\ University of Bern \\ CH-3012 Bern, Switzerland \\ E-mail: deuzemanditp.unibe.ch, wenger@itp.unibe.ch
}

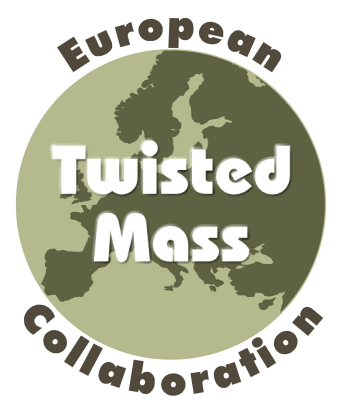

The recently introduced gradient flow suggests a way to obtain normalized gauge field quantities from lattice QCD. It is not only useful to calculate quantities like the renormalized topological susceptibility, but also allows for a very precise scale setting using the renormalized energy density. We will use this approach to investigate these quantities in detail on gauge field ensembles produced with dynamical Wilson twisted mass fermions. Attention will be paid to the impact of lattice artifacts, quark mass and finite volume effects on the determination of the scale.

The 30th International Symposium on Lattice Field Theory

June 24 - 29, 2012

Cairns, Australia

\footnotetext{
* Speaker.
} 


\section{Introduction}

A series of papers from recent years [1-4] examined the properties of gauge fields $U$ as they evolve down the gradient of a pure gauge action $S$ towards their classical minimum. For any given gauge configuration $U$, this defines a curve $V_{t}$ in configuration space parametrized by a fictional flow time $t$ as

$$
\frac{\partial}{\partial t} V_{t}(x, \mu)=-g^{2}\left[\frac{\partial}{\partial_{x, \mu}} S\left(V_{t}\right)\right] V_{t}(x, \mu),
$$

with the boundary condition $V_{0}(x, \mu)=U(x, \mu)$. This differential equation can be integrated numerically and for infinitesimal values of $\varepsilon$ one obtains

$$
V_{t+\varepsilon}(x, \mu)=\exp \left[\varepsilon T^{a} \partial_{x, \mu}^{a} S\left(V_{t}\right)\right] V_{t}(x, \mu),
$$

with $T^{a}$ the generators of SU(3). The expectation value $\left\langle O_{t}\right\rangle$ of any observable $O$ at flow time $t$ is defined as its expectation value on the ensemble of fields $V_{t}$. Any valid pure gauge action $S$ can be chosen, but these proceedings use the standard Wilson plaquette action.

While the curves $V_{t}$ have a range of potential applications, one very practical observation is that they define a reversible and analytical smearing scheme for configurations. For the Wilson plaquette action used here, the numerical integration in this smearing scheme corresponds to a number of infinitesimal stout smearing steps [5]. The dimensionful flow time $t$ plays the rôle of a reference scale and it was shown [4] that the fields $V_{t}$ at finite flow time $t$ are actually renormalized to all orders in perturbation theory, promoting $t$ to a proper renormalization scale.

The gradient flow can be used in any type of analysis for which one would normally require a smearing procedure. There are several applications, however, that rely on the unique properties of the gradient flow method - namely, its reversibility and analyticity. Since the flow trajectory is renormalized, the renormalization group invariant integrated topological susceptibility $V \chi_{T}$ can be determined directly from a calculation of the field theoretical topological charge $Q_{t}=F_{t} \tilde{F}_{t}$, with $F$ the QCD field strength tensor. On the other hand, by monitoring the value of the energy density under the effect of flow equations, the renormalization group flow can provide a measure of the effective physical scale that $t$ represents. In these proceedings, we will describe some results obtained by using the gradient flow on configurations generated using twisted mass Wilson fermions [6] to analyze its potential both for studying the topological properties of these configurations and for its usage in setting the scale of the simulation.

\section{Scale setting methodology}

It has been suggested [2] that an unambiguous scale can be defined from the flow time $t$, using the energy density $E_{t}$. The latter can be defined simply from the plaquette, or from a symmetric discretization of $F$. Both definitions agree in the continuum and we will refer to them as 'naive' and 'symmetrized', respectively. If we find the point where the dimensionless quantity $t^{2} E_{t}$ reaches a given value, e.g. $t_{0}^{2} E_{t_{0}}=0.3$, then $\sqrt{t_{0}}$ is a well-defined gluonic length scale. To use $t_{0}$ for scale setting, it is important to control its lattice artifacts. In fact, it turns out that they can be rather noticeable, as illustrated by the difference between curves based on the naive and symmetrized energy density definitions in figure 1a. Since these artifacts appear mainly at small values of the 


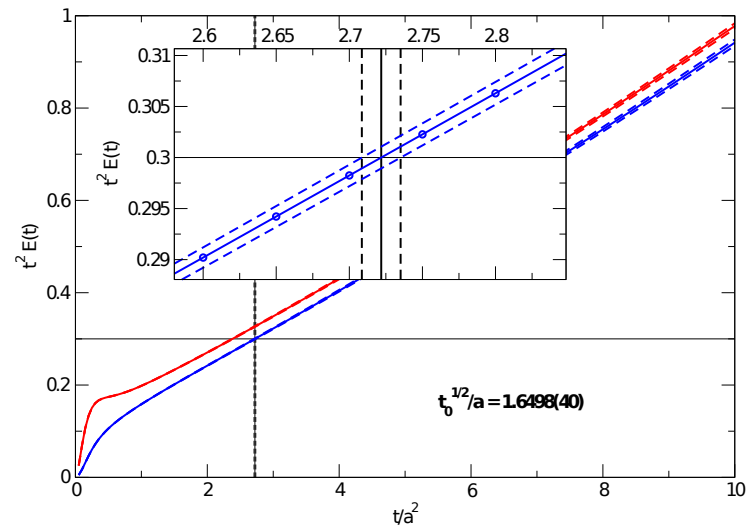

(a) Determination of $\left.t^{2} E_{t}\right|_{t=t_{0}}=0.3$

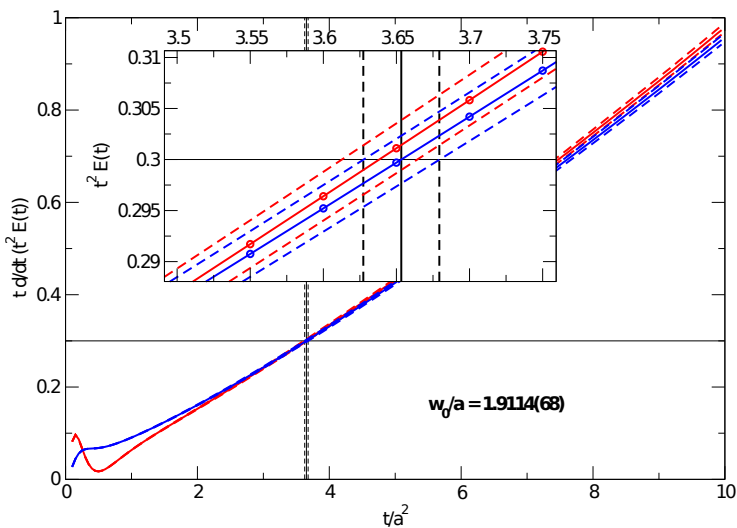

(b) $\left.t \frac{\mathrm{d}}{\mathrm{d} t}\left(t^{2} E_{t}\right)\right|_{t=w_{0}}=0.3$

Figure 1: A graphical comparison of the determination of scales from the gradient flow for an arbitrary configuration with $\beta=1.90$ and a $\mu=0.025: \sqrt{t_{0}}$ on the left, $w_{0}$ on the right. Insets show an enlarged view of the area where the curves hit the reference value of 0.3 for the symmetrized plaquette definition (blue line). The equivalent curve using the naive plaquette definition (red), which should differ by lattice artifacts, deviates enough to not be present in the inset on the left, but is not significantly different on the right.

flow time, they do not necessarily interfere with the usage of the longer distance $\sqrt{t_{0}}$ for scale setting. Their presence does, however, introduce a discretization scheme dependence, such that the measured value of $t_{0}$ will only agree in the continuum limit.

To circumvent this issue, the BMW collaboration [7] proposed to use the quantity

$$
W_{t} \equiv t \frac{\mathrm{d}}{\mathrm{d} t}\left(t^{2} E_{t}\right)
$$

in order to fix the scale. Since this is sensitive to the slope of the energy density, rather than its absolute value, the influence of the lattice artifacts at short flow times will all but vanish at the larger flow times that are of interest for scale setting purposes (see figure 1b). The BMW collaboration defined a reference scale $w_{0}$ through the condition $W_{w_{0}^{2}}=0.3$ and proceeded to determine its value at physical quark masses in the continuum limit, using $m_{\Omega}$ as the physical reference mass. In doing so, they compared two different actions: an $N_{f}=2+1$ staggered action with two levels of stout smearing and an $N_{f}=2+1$ Wilson clover action with two levels of HEX smearing. Both actions showed a nearly flat continuum limit and a consistent extrapolation to $w_{0}=0.1755(18)_{\text {stat }}(04)_{\text {sys }}$.

\section{Lattice scales}

We set out to check the properties of $w_{0}$ using our $N_{f}=2+1+1$ twisted mass ensembles [6] at three distinct values of the lattice gauge coupling $\beta=1.90,1.95$ and 2.10. Our focus is on its potential as a precise physical scale largely unaffected by systematic errors. A first check of its suitability for such an application is given by the measurable effects of finite volume on its value. Here, we reproduced the earlier observation [7] of very minor finite volume effects in $w_{0}$. An explicit comparison of ensembles at differing volumes, but otherwise identical parameters showed effectively negligible differences for values of $m_{\mathrm{ps}} L$ as low as 2.5. Since this value is smaller 
than what is generally considered practical to extract most other observables, it appears that finite volume effects in $w_{0}$ should not generally be a concern.

Another potential issue, affecting the overall precision to which $w_{0}$ could be determined, is the auto-correlation. The energy density is not particularly prone to large auto-correlations per se, but the gradient flow should expose the potentially more correlated physics at longer distances. In fact, as figure 2 demonstrates, correlations tend to be quite high for the action density evaluated at finite flow time. The magnitude of the effect does depend on the details of the ensemble, but appear systematically for all ensembles. The upshot of this is that the small systematic uncertainties in $w_{0}$

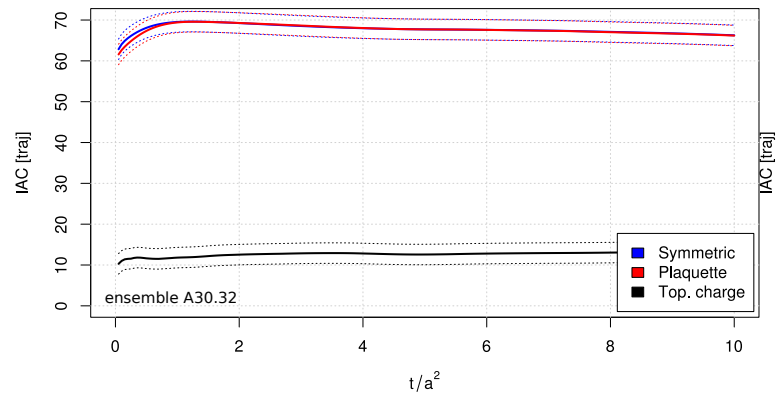

(a) $\beta=1.90, a \mu=0.030, V=32^{3} \times 64$

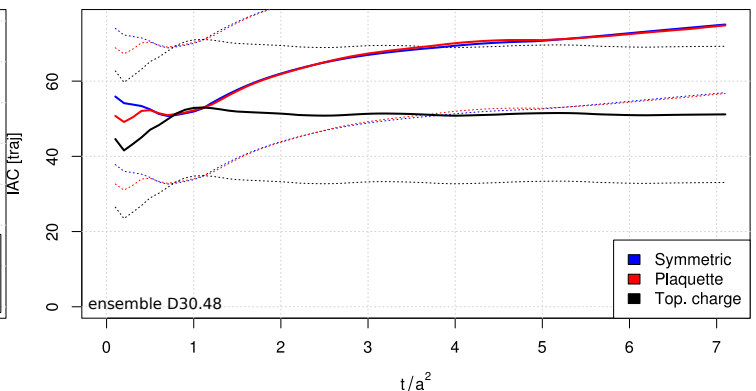

(b) $\beta=2.10, a \mu=0.030, V=48^{3} \times 96$

Figure 2: Integrated auto-correlations (IAC) in units of trajectories measured on two arbitrary ensembles as a function of the flow time for three observables: the plaquette, symmetrized plaquette and topological charge. Auto-correlations tend to rise sharply as the influence of UV scales is suppressed, exposing a much higher degree of correlation than that obtained for the unsmeared observables. Notably, the autocorrelations tend to be at least as large as those found for the topological charge.

could be dominated by underestimated statistical ones. While the determination of $w_{0}$ can be done to very high accuracy on each configuration, as can be seen from inset of figure $1 \mathrm{~b}$, this precision may not translate to a high precision for the ensemble average. In fact, using a standard binning procedure to estimate the true statistical errors, it appears that the inherent precision of $w_{0}$ is not too different from that of $r_{0}$ determined on the same ensemble.

For a mass independent scale setting, it would be preferable to have the value of $w_{0}$ in the chiral limit or at the physical point. In figure 3, we have plotted the values of $w_{0}$ for all ensembles against $m_{\mathrm{ps}}^{4}$, since this was found to give good linear fits for previous extrapolations of $r_{0}$ [6]. To compare data on different lattice scales, values were rescaled by the respective chiral limits of such a fit. Results for $w_{0}$ don't vary drastically, as the maximum relative change in value of $w_{0}$ is less then $6 \%$ over the full range of masses. But given the spread of the points and the size of their errors, there seems to be no monotonous, or even smooth, functional form that would produce a reasonable fit. This, combined with the large auto-correlations described earlier, leads us to conclude that we are most likely underestimating the errors substantially. Either way, using a chiral limit extracted in this way does not seem reasonable. We therefore follow an alternative procedure, performing an NLO SU(2) chiral extrapolation in the pion sector with all quantities expressed in units of $w_{0}$ as measured on each ensemble (figure 4a). This provides a sensible extrapolation while avoiding the large uncertainties from a separate chiral extrapolation of the scale. By determining the physical ratio of the pion decay constant and pion mass squared at each lattice spacing, this procedure allows us to extract the value of $w_{0}$ at the physical point for each lattice spacing separately. 


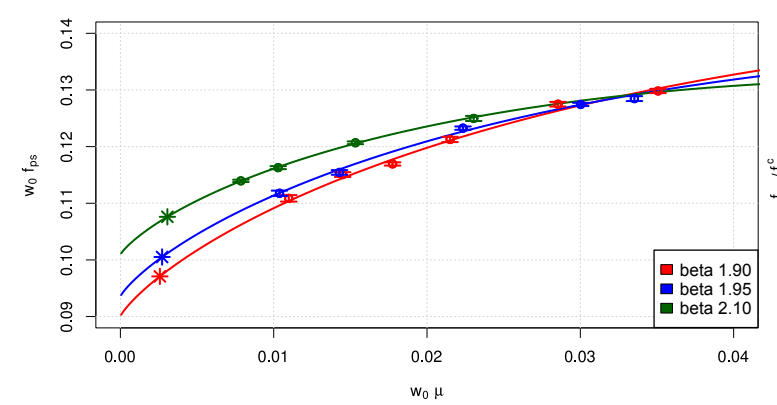

(a) Chiral extrapolation of $w_{0} f_{\mathrm{ps}}$.

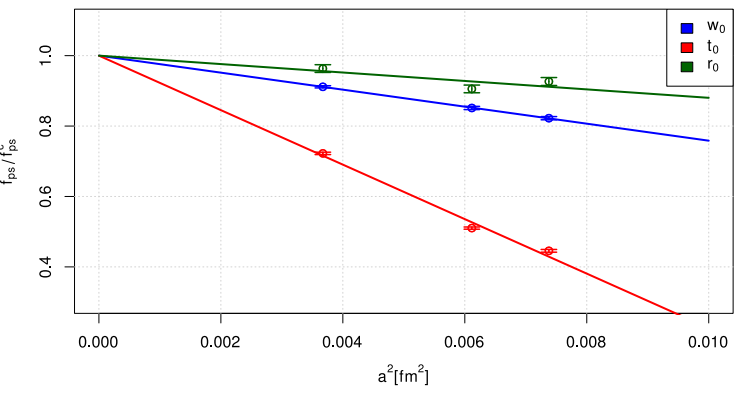

(b) Continuum extrapolation of $w_{0} f_{\mathrm{ps}}$.

Figure 4: (a) The chiral extrapolation of $w_{0} f_{\mathrm{ps}}$ according to $S U(2) \chi_{P T}$ to NLO. A physical value for this quantity (indicated by an asterisk) is extracted by matching to the physical ratio $m_{\mathrm{ps}} / f_{\mathrm{ps}}$. (b) The continuum extrapolation of three lattice scales, as determined from the value of the pion decay constant at the physical point. Results have been rescaled by the continuum extrapolation value $f_{\mathrm{ps}}^{c}$ to allow for a comparison of the relative size of lattice artifacts. The value of the lattice spacing was obtained from the pion mass at the physical point.

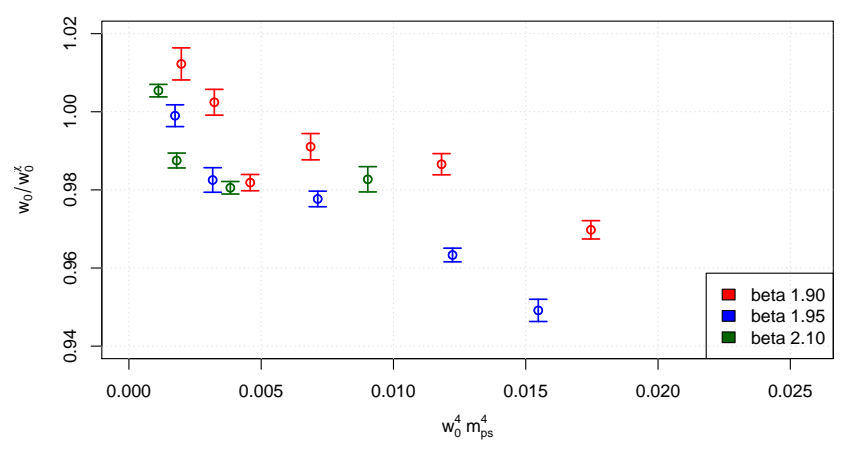

Figure 3: Measurements of $w_{0}$ per lattice spacing, rescaled by the results from linear chiral extrapolation in $m_{\mathrm{ps}}^{4}$ similar to the extrapolation of $r_{0}$ in [6].
Figure 4a shows that the physical values of $w_{0} f_{\mathrm{ps}}$ vary appreciably between the different lattice spacings. The magnitude of these lattice artifacts can be estimated from a continuum extrapolation of $w_{0} f_{\mathrm{ps}}$. We show this in figure $4 b$, together with results from an identical analysis in terms of the scales $\sqrt{t_{0}}$ and $r_{0}$. Since tuning to maximal twist gives our twisted mass fermions an automatic $\mathscr{O}\left(a^{2}\right)$ improvement, a linear extrapolation in the lattice spacing squared should be appropriate to

estimate leading order lattice artifacts. The quality of this extrapolation is good for $w_{0}$, whereas the fits are somewhat poorer for both $r_{0}$ and $\sqrt{t_{0}}$. All extrapolations are rescaled by their continuum value, to expose the deviations on identical scales.

The scale $w_{0}$ was introduced to designed to lack the impact of lattice artifacts found in $\sqrt{t_{0}}$ and it is clear that they are indeed much less pronounced. However, we do not reproduce the near absence of lattice artifacts reported by the BMW collaboration [7]. In fact, our lattice artifacts appear to be smaller for $r_{0}$. The explanation may lie in the differences between the different actions used in both studies. One possible cause could be the absence of smearing in our setup, but factors as diverse as the twisted mass formalism, the use of an Iwasaki gauge action or the absence of clover improvement could all be of influence. The impact of these different factors in general can only be estimated once results using a wider range of actions become available. It is also worth pointing out that the origin of the deviations may even be a more subtle interplay between $w_{0}$ 
and other observables used in our analysis. In this case, though, it is not clear why the otherwise identical analysis using $r_{0}$ would be impacted less.

Using the value of $w_{0} f_{\mathrm{ps}}$ determined in the continuum limit to obtain a physical value for $w_{0}$, gives a preliminary value of $w_{0}=0.1782 \mathrm{fm}$. The current analysis is not sufficiently sophisticated to provide a reliable error estimate on this number, but since it already lies within $1.5 \sigma$ of the value of $w_{0}=0.1755(18)_{\text {stat }}(04)_{\text {sys }}$ fm provided by the BMW collaboration, it should be consistent with it.

\section{Topological susceptibility}

The gradient flow allows also for an efficient calculation of the topological susceptibility $\chi_{T}$ via a measurement of $F_{t} \tilde{F}_{t}$ on the evolved lattices. This particular method of calculation should provide the benefit that, through the renormalized nature of the field for any $t \neq 0$, we should get immediate access to the renormalization group invariant observable $V \chi_{T}$. This seems to be born out by the behavior of the observable as a function of the flow time shown in figure $5 \mathrm{a}$. Once the gradient flow has progressed sufficiently far, $\chi_{T}$ becomes independent of the flow time, i.e. of the renormalization scale.

We plotted our measurements for $\chi_{T}$ for a number of ensembles at our two finest lattice spacings versus the pion mass squared in figure $5 \mathrm{~b}$. A suppression of the topological susceptibility

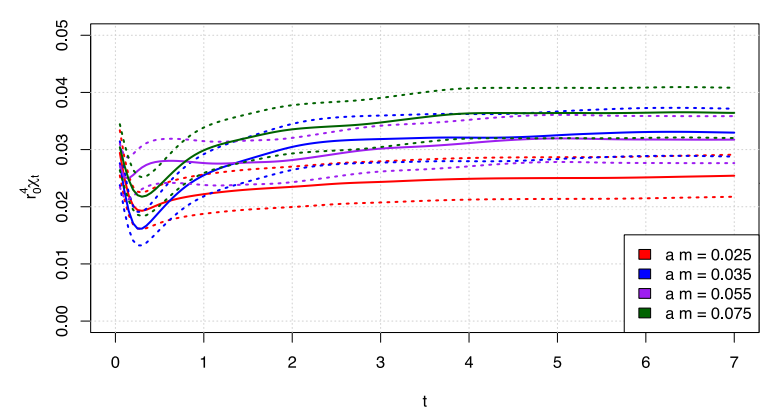

(a) $\chi_{T}$ versus flow time.

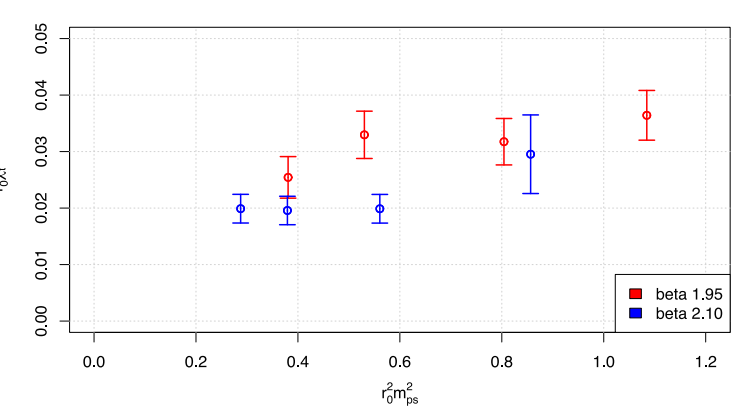

(b) $\chi_{T}$ versus the pion mass squared.

Figure 5: The value of $r_{0} \chi_{T}$ calculated from $F_{t} \tilde{F}_{t}$ on $V_{t}$. (a) As a function of the flow time for various bare quark masses at $\beta=1.95$ at fixed lattice volume. (b) As a function of the pion mass squared for two values of $\beta$.

with decreasing pion mass is expected, but this is not yet obvious from the current data. It should be noted that the errors are still large enough to allow for a downwards trend, even while autocorrelations may be underestimated. There may also still be remnant lattice artifacts influencing these data. If so, those could be partly disentangled through measurements of $\chi_{T}$ by using a different methodology, such as in [8]. In order to enhance discriminatory power of the current results, it would be beneficial to implement variance reduction methods such as those used in [9]. It would also be very valuable to obtain additional data closer to the chiral limit.

\section{Conclusions}

The gradient flow method is a continuous and invertible scheme for smoothing configurations, 
based upon a first order homogeneous differential equation of the gauge action. We have implemented and tested the gradient flow method on a range of $N_{f}=2+1+1$ twisted mass Wilson fermion ensembles produced by the ETM Collaboration [6].

As a first practical application of the gradient flow, we tested its use in scale setting, focusing on the $w_{0}$ scale recently proposed by the BMW collaboration [7]. While this scale can be determined precisely and unambiguously on a configuration by configuration basis, the gradient flow procedure exposed large auto-correlations in the energy density, implying that the statistical errors are easily underestimated in these quantities and subsequently in $w_{0}$.

The use of $w_{0}$ instead of $t_{0}$ appears to dramatically decrease discretization artifacts, but they do remain noticeable. This differs from the findings of the BMW collaboration for both fermion actions discussed. The cause of this discrepancy cannot be determined from present data alone. It may rest in the difference between the actions used or, with lesser likelihood, be somehow introduced through another observable used in our analysis. A continuum extrapolation using $f_{\pi}$ to set the scale, however, produces a value consistent with the value of $w_{0}$ found by the BMW configuration using the $\Omega$ mass as the physical scale.

Our attempts to use the renormalizing properties of the gradient flow to extract estimates of the topological susceptibility $\chi_{T}$ met with success. The average of $\chi_{T}$ stabilizes after a short flow time for all ensembles studied. The current results are not yet sufficient to draw any firm conclusions on the behavior of $\chi_{T}$ towards the chiral limit. This situation could be probably be improved by the implementation of a variance reduction scheme, as well as additional data towards the chiral limit.

\section{References}

[1] M. Lüscher, Trivializing maps, the Wilson flow and the HMC algorithm, Commun. Math. Phys. 293 (2010) 899.

[2] M. Lüscher, Properties and uses of the Wilson flow in lattice QCD, JHEP 1008 (2010) 071.

[3] M. Lüscher, Topology, the Wilson flow and the HMC algorithm, PoS LATTICE 2010 (2010) 015.

[4] M. Lüscher and P. Weisz, Perturbative analysis of the gradient flow in non-abelian gauge theories, JHEP 1102 (2011) 051.

[5] C. Morningstar and M. J. Peardon, Analytic smearing of SU(3) link variables in lattice QCD, Phys. Rev. D 69 (2004) 054501.

[6] R. Baron et al., Light hadrons from lattice QCD with light $(u, d)$, strange and charm dynamical quarks, JHEP 1006 (2010) 111.

[7] S. Borsanyi et al., High-precision scale setting in lattice QCD, JHEP 1209 (2012) 010.

[8] K. Cichy et al. Topological susceptibility and chiral condensate with $N_{f}=2+1+1$ dynamical flavors of maximally twisted mass fermions, PoS LATTICE 2011 (2011) 102.

[9] A. Bazavov et al., Topological susceptibility with the asqtad action, Phys. Rev. D 81 (2010) 114501. 